\title{
$\Lambda$ photoproduction studied with an electromagnetic calorimeter FOREST
}

\section{Yusuke Tsuchikawa*}

Research Center for Electron Photon Science (ELPH), Tohoku University, Sendai 982-0826,

Japan

E-mail: tsuchikawadins.tohoku.ac.jp

\section{H. Fujimura ${ }^{1} a$, H. Fukasawa ${ }^{1}$, R. Hashimoto ${ }^{1 b}$, T. Ishikawa ${ }^{1}$, T. Iwata ${ }^{2}$, S. Kaida ${ }^{1}$,} J. Kasagi ${ }^{1}$, A. Kawano ${ }^{3}$, S. Kuwasaki ${ }^{1}$, K. Maeda ${ }^{4}$, S. Masumoto ${ }^{5}$, M. Miyabe ${ }^{1}$, F. Miyahara ${ }^{1 c}$, K. Mochizuki ${ }^{1}$, A. Nakamura ${ }^{1}$, K. Nawa $^{1}$, S. Ogushi ${ }^{1}$, Y. Okada ${ }^{1}$, Y. Onodera' ${ }^{1}$, K. Ozawa ${ }^{6}$, Y. Sakamoto ${ }^{3}$, M. Sato ${ }^{1}$, H. Shimizu ${ }^{1}$, H. Sugai ${ }^{1}$, K. Suzuki ${ }^{1 d}$, Y. Tajima ${ }^{2}$, S. Takahashi ${ }^{1}$, H. Yamazaki ${ }^{1}$, and H.Y. Yoshida ${ }^{2}$

${ }^{1}$ Research Center for Electron Photon Science (ELPH), Tohoku University, Sendai 982-0826, Japan

${ }^{2}$ Department of Physics, Yamagata University, Yamagata 990-8560, Japan

${ }^{3}$ Department of Information Science, Tohoku Gakuin University, Sendai 981-3193, Japan

${ }^{4}$ Department of Physics, Tohoku University, Sendai 980-8578, Japan

${ }^{5}$ Department of Physics, University of Tokyo, Tokyo 113-0033, Japan

${ }^{6}$ Institute of Particle and Nuclear Studies, KEK, Tsukuba 305-0801, Japan

a Present address: Department of Physics, Wakayama Medical University, Wakayama 641-8509, Japan

${ }^{b}$ Present address: Department of Physics, Yamagata University, Yamagata 990-8560, Japan

${ }^{c}$ Present address: Accelerator Laboratory, KEK, Tsukuba 305-0801, Japan

${ }^{d}$ Present address: Technical Support Division, Osaka University, Toyonaka 560-0043, Japan

\begin{abstract}
Baryon resonances have been experimentally studied by means of meson photoproduction experiments at Research Center for Electron Photon Science (ELPH), Tohoku University. Photons from neutral meson decays are detected with an electromagnetic calorimeter complex FOREST. The analysis of the events proceeds for photoproduction of single neutral mesons which decay into several photons. To extend the analysis to the events having several charged particles, the $\gamma p \rightarrow K^{+} \Lambda$ reaction is investigated at the beginning.
\end{abstract}

Sixth International Conference on Quarks and Nuclear Physics,

April 16-20, 2012

Ecole Polytechnique, Palaiseau, Paris

\footnotetext{
* Speaker.
} 


\section{Introduction}

Baryon spectroscopy is an important testing ground for understanding the properties and interactions of the strong interacting matter at low energies. At Research Center for Electron Photon Science (ELPH), baryon resonances are studied by meson photoproduction experiments [山, 口] with an electromagnetic (EM) calorimeter complex FOREST [3]. The incident photon energy ranging from 550 to $1150 \mathrm{MeV}$ is tagged [䧃], which corresponds to the baryon mass from 1380 to 1740 MeV. FOREST consists of three different calorimeters: 192 pure CsI crystals, 252 lead scintillating fiber modules, and 62 lead glass Čerenkov counters. The energy resolutions of the calorime-

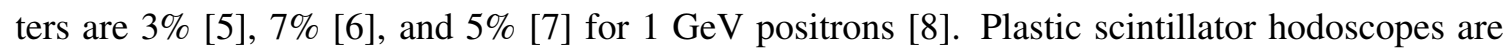
placed in front of each calorimeter to identify the charge of the incident particle. Figure $\mathbb{W}$ shows the schematic view of FOREST. The baryon that decays into $\pi^{0} N$ or $\eta N$ are intensively investigated since these neutral mesons decay into two photons. The response of FOREST detectors are well understood for photons, and the analysis of the single $\pi^{0}$ and $\eta$ photoproduced events proceeds $\left[Q,\left[\begin{array}{l}0 \\ ]\end{array}\right.\right.$. To extend the analysis to the events having several charged particles, the $\gamma p \rightarrow K^{+} \Lambda$ reaction is investigated at the begining.

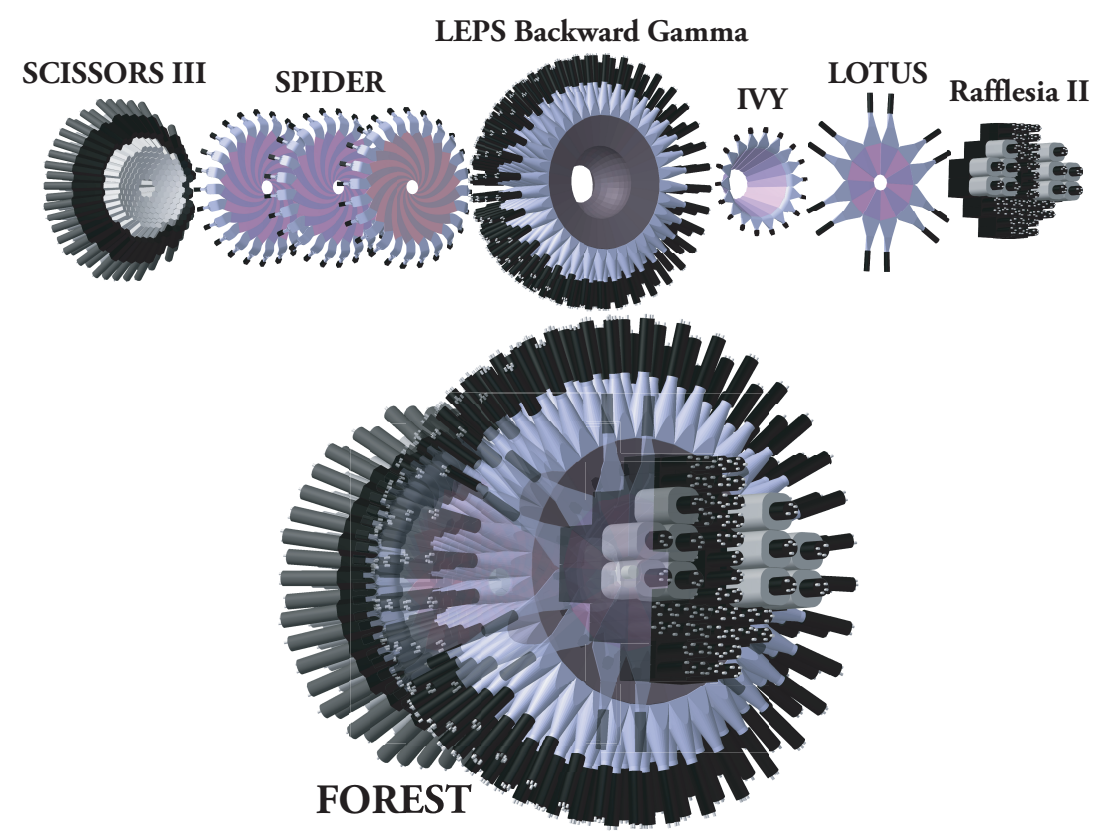

Figure 1: Schematic view of FOREST. It consists of three EM calorimeters: the forward one with 192 pure CsI crystals 'SCISSORS III (S3),' the central one with 252 lead scintillating fiber modules 'LEPS Backward Gamma detector (BG),' and the backward one with 62 lead glass Čerenkov counters 'Rafflesia II (R2).' Plastic scintillator hodoscopes are placed in front of these calorimeters: SPIDER, IVY, and LOTUS.

\section{Precise calibration of hodoscopes}

The $\gamma p \rightarrow K^{+} \Lambda \rightarrow K^{+} \pi^{-} p$ events have three charged particles in the final state. It is very challenging to distinguish these charged particles without the momentum analysis by the magnetic 
field. The preceise energy and timing measurements are required for plastic scintillator (PS) hodoscopes. The emitted scintillation photons in a PS are read out at the single end by a photomultiplier tube (PMT) Hamamatsu R8900U. Since the acceptance of them depends on the distance from its cathod window, the energy measured by a PMT is as a function of the distance. The measured timing is also as a function of the distance (the path length of the scintillation photons) because the propagation of the photons in a PS takes a finite time. Since we adopt a leading edge type discriminator module for the generation of the logic signal, the measured timing walks according to the pulse-height of the signal.

At first, the energy distributions for the minimum ionizing particles are estimated at the different path lengths for all the PS's. The path length is given by the distance from the photon beam axis for SPIDER PS's and LOTUS PS's, and given by the polar angle for IVY PS's. The energy (stopping power) is reconstructed by using the peak position as a function of the path length so that it should be 1 for the minimum ionizing particles.

The time difference distributions between PS and calorimeter modules for the particles traveling at the speed of light are estimated at the different path lengths for all the PS's. Two clusters generated by the EM shower are selected (a large energy leakage exists from the central module), the average timing of them are used for the origin for the timing measurement. Figure $\square$ shows the mean of the timing difference distribution as a function of the measured energy for different path lengths (polar angles) for IVY PS's. The polar angle is determined by the corresponding cluster behind the PS. The reosolution of the measured time difference is about 300-400 ps.

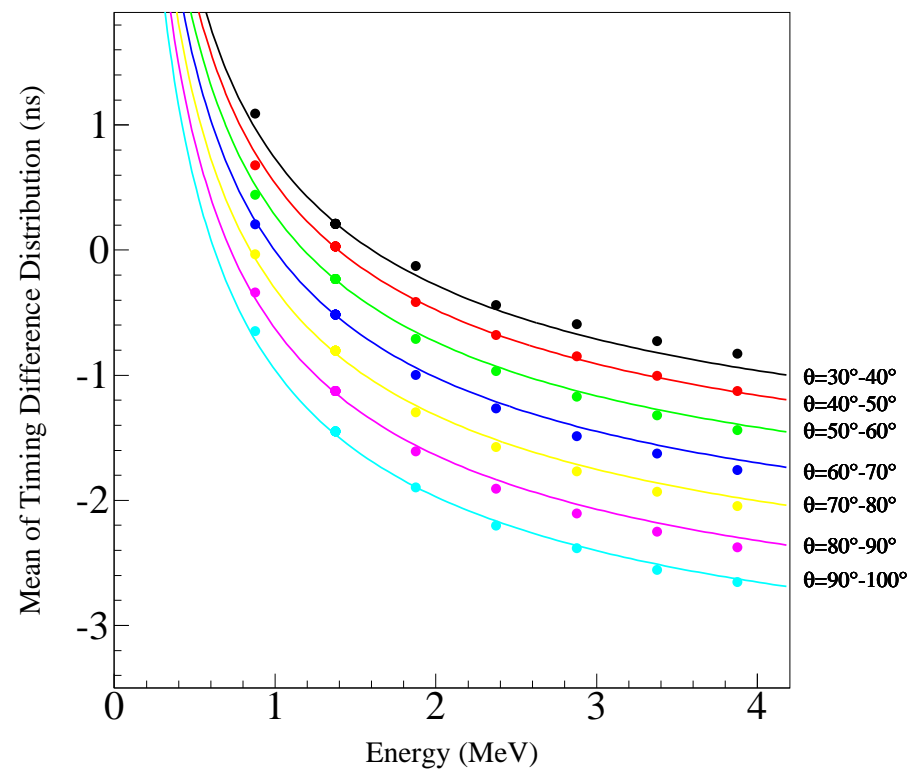

Figure 2: Mean of the timing difference distirbution as a function of the measured energy for different path lengths of scintillation photons (polar angles) for IVY PS's. The color indicates the polar angle of the particle. 


\section{Selection of $\Lambda$ produced events}

The $\gamma p \rightarrow K^{+} \Lambda$ events are selected where $\Lambda$ decays into $\pi^{-}$and $p$. For the simplicity, we focus on the events that $\pi^{-}$and $p$ are detected with the forward detector S3 and the other $K^{+}$is detected with the central detector BG since BG consists of sampling calorimeter modules and its energy reoslution for charged hadrons is poor. At first, the $\pi^{-}$and $p$ candidate clusters are selected by using the $\Delta E-E$ correlation. The high energy protons penetrate the CsI crystals of S3, and some events exist that two charged pion clusters misidentified as $\pi^{-}$and $p$. Figure Bla) shows the time diffence between the $\pi^{-}$and $p$ candidate clusters. To reject the events having two charged pion clusters, the time difference is required to be larger than $1 \mathrm{~ns}$. Since the proton in the initial state is at rest, the emitted azimuthal angles are opposite between $\pi^{-} p$ and $K^{+}$. Figure Bb) shows the difference of the azimuthal angles between $\pi^{-} p$ and $K^{+}$. To select three clusters as a candidate for $K^{+}$and $\pi^{-} p$ from $\Lambda$ decay, the difference of azimuthal angles is limited from 150 to 210 degrees.
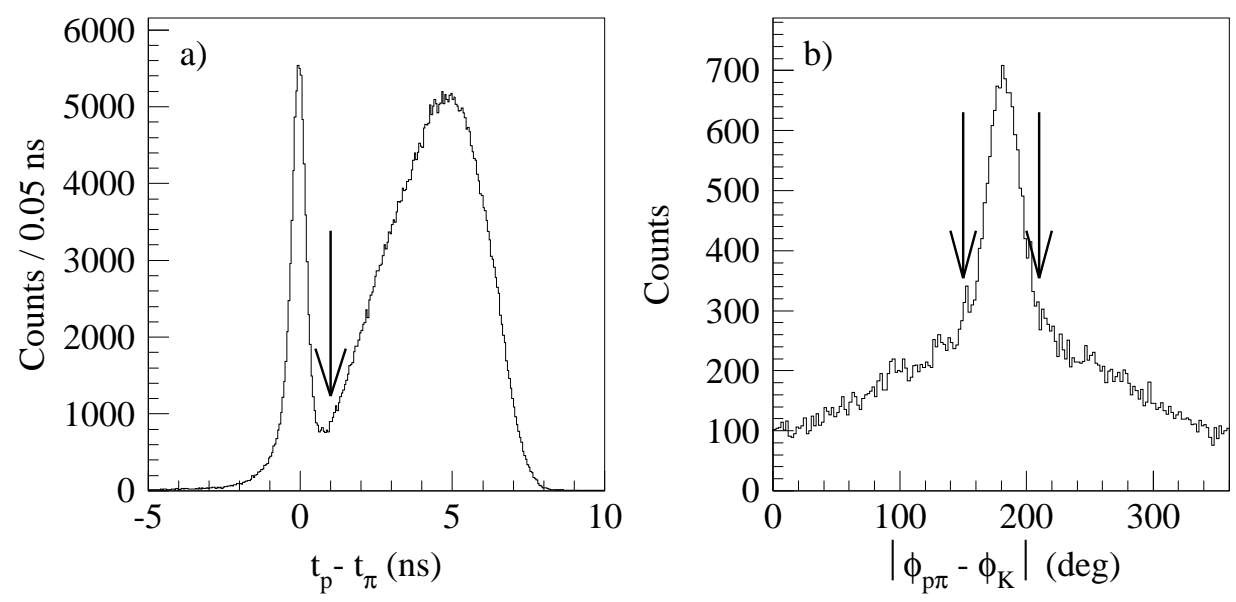

Figure 3: a) Time diffence between the $\pi^{-}$and $p$ candidate clusters. The cut point is indicated by the arrow to extract the $\pi^{-}$and $p$ candidates. b) Difference of the azimuthal angles between $\pi^{-} p$ and $K^{+}$. The cut points are indicated by the arrows to select the events that $\pi^{-} p$ and $K^{+}$are emitted in the opposite directions.

Now, we have the events having three clusters as a candidate for the $\gamma p \rightarrow K^{+} \Lambda$ reaction. Figure $\mathbb{\theta}$ shows the $\pi^{-} p$ invariant mass distributions for $E_{\gamma} \geq 911 \mathrm{MeV}$ and for $E_{\gamma}<911 \mathrm{MeV}$. The incident energy of $911 \mathrm{MeV}$ corresponds to the threshold for the $\gamma p \rightarrow K^{+} \Lambda$ reaction. The $\Lambda$ peak is clearly observed only for the higher energy region of $E_{\gamma}$.

\section{Summary}

Meson photoproduction experiments were carried out with an EM calorimeter complex FOREST at ELPH to study baryon resonances. The preceise energy and timing calibration was made for PS hodoscopes where the scintillation photon path length and pulse-height time-walk are taken into account. The $\gamma p \rightarrow K^{+} \Lambda \rightarrow K^{+} \pi^{-} p$ events having three charged particles in the final state were examined after the calibration. The $\Lambda$ peak in the $\pi^{-} p$ invariant mass distribution is clearly observed above the reaction threshold of the incident photon energies. 

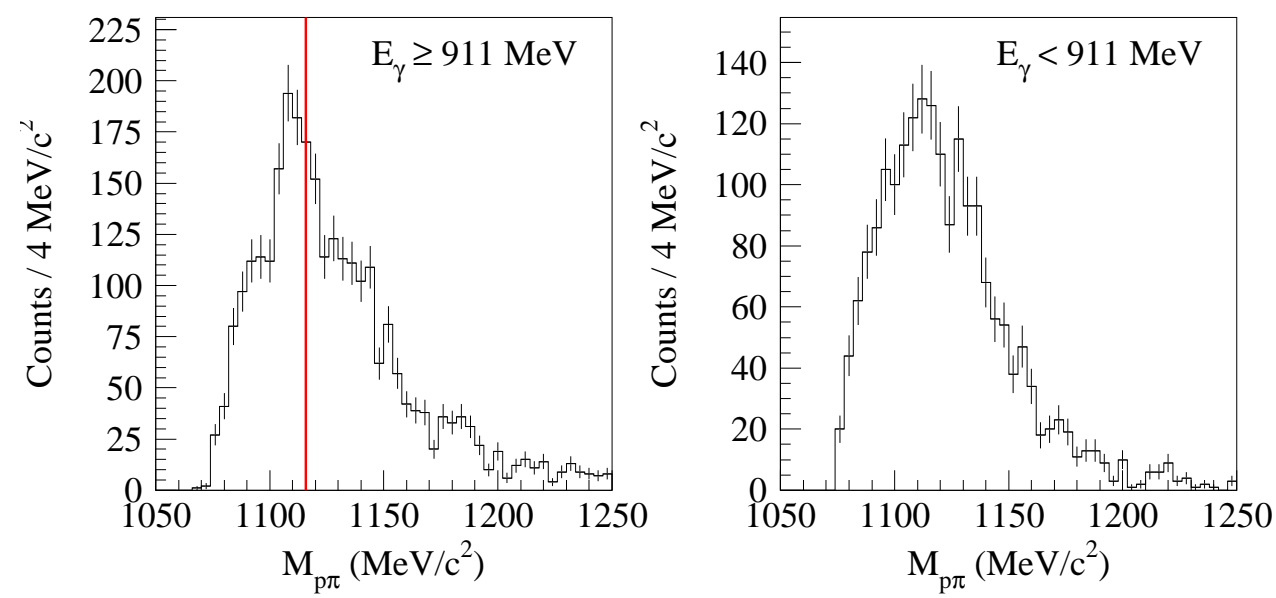

Figure 4: $\pi^{-} p$ invariant mass distributions for $E_{\gamma} \geq 911 \mathrm{MeV}$ (the left panel) and for $E_{\gamma}<911 \mathrm{MeV}$ (the right panel). The red line indicates the rest mass of $\Lambda$.

\section{References}

[1] T. Nakabayashi et al., Phys. Rev. C 74, 035202 (2006).

[2] T. Kinoshita et al., Phys. Lett. B 639, 429 (2006).

[3] T. Ishikawa, Int. J. Mod. Phys. E 19, 2393 (2010);

K. Suzuki et al., Mod. Phys. Lett. A 24, 978 (2009).

[4] T. Ishikawa et al., Nucl. Instr. and Meth. 622, 1 (2010).

[5] A. Nakamura, T. Ishikawa, Y. Tsuchikawa et al., Research Report of LNS 42\&43, Tohoku University, 75 (2011);

H. Sugai, T. Ishikawa et al., Research Report of LNS 42\&43, Tohoku University, 88 (2011).

[6] K. Mochizuki, T. Ishikawa et al., Research Report of LNS 40, Tohoku University, 15 (2008).

[7] T. Ishikawa et al., Research Report of LNS 42\&43, Tohoku University, 82 (2011);

M. Sato, T. Ishikawa et al., Research Report of LNS 41, Tohoku University, 22 (2009).

[8] T. Ishikawa et al., Research Report of LNS 40, Tohoku University, 6 (2008);

Y. Tsuchikawa, T. Ishikawa et al., ELPH Annual Report 1, 107 (2010).

[9] T. Ishikawa, talk at ECT* Workshop on Strange Hadronic Matter (2011).

[10] T. Ishikawa, Y. Tsuchikawa et al., ELPH Annual Report 1, 1 (2010). 